\title{
The role of serological testing for Chlamydia trachomatis in differential diagnosis of pelvic pain
}

\author{
Beata Łój', Agnieszka Brodowska², Sylwester Ciećwież², Iwona Szydłowska², Jacek Brodowski², \\ Marek Łokaj ${ }^{4}$, Andrzej Starczewski ${ }^{2}$ \\ ${ }^{1}$ Frauenklinik Asklepios Klinkum Uckermark, Schwedt, Germany \\ ${ }^{2}$ Departament of Gynecology and Urogynecology, Pomeranian Medical University, Szczecin, Poland \\ ${ }^{3}$ Primary Healthcare Center, Pomeranian Medical University, Szczecin, Poland \\ ${ }^{4}$ Clinic of Plastic, Endocrine and General Surgery, Pomeranian Medical University, Szczecin, Poland
}

Łój B, Brodowska A, Ciećwież S, Szydłowska I, Brodowski J, Łokaj M, Starczewski A. The role of serological testing for Chlamydia trachomatis in differential diagnosis of pelvic pain. Ann Agric Environ Med. 2016; 23(3): 506-510. doi: 10.5604/12321966.1219196

\begin{abstract}
Introduction. Pelvic pain is typically associated with pelvic inflammatory disease (PID). The most common cause of PID is Chlamydia trachomatis. The aim of this study was to verify the role of serological testing for Chlamydia trachomatis in patients with suspected PID.

Materials and method. The retrospective study included 185 patients with pelvic pain hospitalized at the Department of Obstetrics and Gynecology in 2003 and 2004. Titers of anti-Chlamydia trachomatislgG and IgA were measured by means ELISA immunoassays. Erythrocyte sedimentation rate (ESR), serum concentration of C-reactive protein (CRP) and leukocyte count (WBC) were also determined. Final diagnosis was established on the basis of laparoscopic examination.

Results. The presence of anti-Chlamydia trachomatis antibodies correlated significantly with abnormal values of ESR, WBC and CRP. The most common laparoscopic pathology were pelvic adhesions, typically found in women with elevated titers of anti-Chlamydia trachomatis IgG.

Conclusion. Serological examination for Chlamydia trachomatis is helpful in evaluation of patients with suspected PID. Elevated titers of anti-Chlamydia trachomatis antibodies are frequently associated with laparoscopic evidence of pelvic adhesions and inflammation.
\end{abstract}

\section{Key words \\ Chlamydia trachomatis, laparoscopy, pelvic pain}

\section{INTRODUCTION}

Pelvic pain is a common complaint of women referred to gynecological practices [1]. Typically, it is associated with pelvic inflammatory disease (PID), especially in young women of reproductive age. PID is not a synonym for pelvic pain, and is the most common cause of pain in the pelvis in young women of reproductive age.

Inappropriate diagnosis is often associated with delayed treatment which increases the risk of serious complications. A clinical trial or examination is neither sensitive nor specific and therefore have little diagnostic value. The most common cause of PID is Chlamydia trachomatis; a total of 15 serotypes (immunotypes) of this pathogen have been identified thus far as an etiological factor of various pathological conditions $[2,3,4]$. Serotypes from L1 and L3 play a role in the etiology of lymphogranuloma venereum (LGV), a nodal or systemic infection, also known as Durand-Nicolas-Favre disease. Systemic LGV typically begins in the urogenital mucosa and spreads via the lymphatics. Its initial manifestations include urethritis, cervicitis, painless buboes and ulcers in the genital area. Lymphadenitis is then observed, along with inguinal redness and swelling, fever, headache, meningeal irritation, conjunctivitis, rash, nausea, vomiting and arthralgia. The last

Address for correspondence: Sylwester Ciećwież, Departament of Gynecology and Urogynecology, Pomeranian Medical University, Szczecin, Poland

E-mail: sylwester.ciecwiez@pum.edu.pl

Received: 20 January 2016; accepted: 30 May 2016 stage of the infection is associated with lower limb and groin swelling [5]. Serotypes from D to K may cause infections of the urogenital tract, eye, pharynx and rectum, reactive arthritis, vascular pathologies and rarely, respiratory and biliary infections. Most of these conditions are transmitted sexually.

Infection with Chlamydia trachomatis stimulates humoral response and synthesis of $\operatorname{IgG}, \operatorname{IgA}$ and $\operatorname{Ig} M$ antibodies by plasmatic cells. Progressive increase in serum concentrations of specific IgM, IgA and IgG is typically observed between 5-20 days of infection. IgM, a marker of acute infection, can be detected about the day 5, and elevated levels of IgA are usually observed after ca. 10 days. Initially, a simultaneous increase in serum concentrations of $\operatorname{IgM}$ i $\operatorname{IgA}$ can be noted. IgG are usually detected at 2-3 weeks of the primary infection. Reinfection is associated with a rapid increase in IgG titer which remains elevated for weeks and then gradually decreases [2].

A number of risk factors for Chlamydia trachomatis infection have been identified to-date. The principal risk factors include young age, female gender, Caucasian race and lack of a spouse $[2,3,4]$. About $40 \%$ of all infections occur in female Caucasians between 15-19 years of age [2, 4]. History of PID is associated with increased risk of reinfection due to persistent structural and functional changes of the uterine tubes, which result in attenuated local and systemic immune response [6]. The risk of infection can be reduced with mechanical methods of contraception, such as condoms [7]. 
Pregnancy also constitutes an important risk factor for Chlamydia trachomatis infection. Chlamydial antigens are found in cervical swabs from up to $5 \%$ of all pregnant women, and many researchers claim a pivotal role of Chlamydia trachomatis infection in the etiology of post-partum and postmiscarriage endometritis [2]. Therefore, all pregnant women are obligatorily examined for the presence of Chlamydia trachomatis to reduce the incidence of perinatal infections with this pathogen. Screening includes examination of cervical swabs in the first and third trimester, as well after each episode of preterm uterine contractions [3,8].

According to many authors, Chlamydia trachomatis infection is associated with an about 5-7-fold increase in the risk for ectopic pregnancy and resultant miscarriage [3]. A link between chlamydial infection and endometriosis has also been postulated [9]. Another consequence of infection with Chlamydia trachomatis may be hepatic capsulitis with phrenic nerve irritation and pain below the right shoulder blade;additionally, the so-called 'violin string' adhesions of the parietal peritoneum to the liver may be found on laparoscopy [10]. Chlamydia trachomatis infection is also associated with increased risk of cervical intraepithelial neoplasia (CIN), and therefore plays an important role in the etiology of cervical squamous cell carcinoma. Patients infected with serotype $G$ are probably six times more likely to develop this malignancy; also infections with chlamydial serotypes I and D are associated with increased risk for neoplasia [11]. Another potential complication of Chlamydia trachomatis infection is reactive arthritis [12].

Diagnosis of PID caused by Chlamydia trachomatis is often challenging due to its heterogeneous symptomatology. In about $80 \%$ of the cases, the infection is asymptomatic. Also many mild infections remain undetected as both patients and their physicians may ignore the symptoms, such as spotting, dyspareunia or vaginal discharge. Diagnostic criteria of chlamydial infection include the presence of abdominal pain, yellowish vaginal discharge, uterine and adnexal tenderness during bimanual vaginal examination whenever pregnancy and other potential causes were excluded [2]. Other, less specific symptoms include fever, elevated erythrocyte sedimentation rate (ESR) and increased serum concentration of C-reactive protein (CRP), as well as a positive result of microbiological examination and the evidence of distended inflamed uterine tubes on transvaginal Doppler. However, both the specificity and sensitivity of clinical signs and symptoms are very low [2].

Microbiological tests play a pivotal role in the diagnostics of Chlamydia trachomatis infection. Other methods, suitable for a direct detection of even slight amount of chlamydial antigens, are immunofluorescence and immunoenzymatic tests with monoclonal antibodies. The group of new generation highly sensitive and highly specific molecular tests, also referred to as nucleic acid amplification techniques (NAAT), include polymerase chain reaction (PCR) and ligase chain reaction (LCR). Finally, infection with Chlamydia trachomatis can be confirmed serologically $[2,3]$.

Treatment of infection of chlamydiatrachomatis has to last at least ten days. Antibiotics of choice, due to strong penetration to the interior cells, are tetracycline, doxycycline, best-tolerated at a dose of $2 \times 100 \mathrm{mg}$ or $2 \times 200 \mathrm{mg}$ per day. Alternatively, quinolones (np.ciprofloksacyne $2 \times 250 \mathrm{mg}$ ), lincosamides (clindamycin $4 \times 300 \mathrm{mg}$ ) orsulfonamides can be prescribed. In pregnancy, macrolides e.g., erythromycinorroxithromycin $4 \times 500 \mathrm{mg} 1 \times 300 \mathrm{mg}$, are recommended. There are an increasing number of reports about the growing resistance to azithromycin and caution is recommended in ist use. There are new reports of the efficacy ryfamipycyny. In cases of chronic or recurrent infection, treatment should last for about twenty days. An important part of the therapy is the treatment of sexual partners. Sexual contact within the six months preceding the appearance of symptoms should be taken into account $[2,3]$.

The aim of this study was to verify the role of serological testing for Chlamydia trachomatis in patients with suspected PID, and to compare the diagnostic value of serology with that of laboratory parameters: ESR, serum CRP concentration and leukocyte count (WBC). Associations between elevated serum titers of anti-Chlamydia trachomatis antibodies and laparoscopic evidence of various pelvic pathologies were also analyzed.

\section{MATERIALS AND METHOD}

The retrospective study included 185 patients with pelvic pain hospitalized at the Department of Obstetrics and Gynecology in Schwedt in 2003 and 2004. History taking included obstetrical and gynecological data (day of menstrual cycle, age at menarche, parity, number of spontaneous miscarriages and abortions, contraceptive method), presence of any systemic diseases, severity of pelvic pain and occurrence of additional symptoms, such as diarrhea or dysuria. Physical examination comprised of bimanual vaginal examination and ultrasonography (Tab. 1).

Table 1. Gynecological and obstetrical characteristics of the analyzed groups

\begin{tabular}{lcccccccc}
\hline \multirow{2}{*}{ Variable } & \multicolumn{2}{c}{$\begin{array}{c}\text { Group A } \\
(\mathrm{n}=115)\end{array}$} & \multicolumn{2}{c}{$\begin{array}{c}\text { Group B } \\
(\mathrm{n}=8)\end{array}$} & \multicolumn{2}{c}{$\begin{array}{c}\text { Group C } \\
(\mathrm{n}=29)\end{array}$} & \multicolumn{2}{c}{$\begin{array}{c}\text { Group D } \\
(\mathrm{n}=33)\end{array}$} \\
\cline { 2 - 10 } & mean & range & mean & range & mean & range & mean & range \\
\hline Age & 28.44 & $20-66$ & 32.25 & $23-53$ & 32.59 & $15-50$ & 29.09 & $16-52$ \\
\hline Age at menarche & 12.62 & $9-17$ & 13.38 & $12-14$ & 12.59 & $9-16$ & 13.27 & $11-17$ \\
\hline Parity $(\mathrm{n})$ & 0.9 & $0-5$ & 1.36 & $0-3$ & 1.38 & $0-3$ & 1.15 & $0-4$ \\
\hline Miscarriages $(\mathrm{n})$ & 0.17 & $0-4$ & 0.13 & $0-1$ & 0.21 & $0-2$ & 0.06 & $0-1$ \\
\hline Abortions $(\mathrm{n})$ & 0.35 & $0-7$ & 0.5 & $0-1$ & 0.59 & $0-7$ & 0.21 & $0-1$ \\
\hline Cycle day & 16.86 & $1-60$ & 18.13 & $10-28$ & 16.03 & $1-32$ & 17.24 & $1-36$ \\
\hline
\end{tabular}

The list of determined laboratory parameters included complete blood count, urinalysis, ESR, CRP and serological testing, namely immunoenzymatic ELISA (MEDAC) for anti-Chlamydiatrachomatis IgA and IgG. ESR $>10 \mathrm{~mm}$ /hour, $\mathrm{WBC}>10,000$ cells $/ \mu$ land serum CRP $>4 \mathrm{mg} / 1$ (i.e. the values exceeding the upper reference limits used at the KlinikumUckermark) were considered abnormal. Antibody titers were considered elevated whenever they exceeded 1:50. In all the patients, final diagnosis was established on the basis of laparoscopic examination. The main indication for laparoscopy were strong pain in the pelvic independently of the other clinical parameters. The aim of the study was laparoscopic confirm or exclude a diagnosis clinically.

The examined women were grouped according to the type of anti-Chlamydia trachomatis antibodies detected and the result of laparoscopy. Four groups of patients were identified based on the seroprevalence of specific antibodies against Chlamydia 
trachomatis: A) with normal $\operatorname{IgA}$ and $\operatorname{IgG}$ titers $(\mathrm{n}=115)$, B) with elevated IgA titers and normal IgG titers $(n=8), C)$ with elevated IgG titers and normal IgA titers $(\mathrm{n}=29)$, and D) with elevated both IgA and IgG titers $(n=33)$. Each group was then stratified based on the normal result of diagnostic laparoscopy or presence of any pathologies, such as pelvic fluid collections and hyperaemia, pelvic adhesions, retroperitoneal pelvic endometriosis, ectopic pregnancy or appendicitis.

Distributions of quantitative variables were analyzed with Shapiro-Wilk test. Mean values of continuous variables were compared with non-parametric tests: Mann-Whitney U-test and Kruskal-Wallis test. Distributions of qualitative variables were compared with chi-square test and Fischer exact test, and the power of relationship between pairs of the variables was determined on the basis of Phi and Cramer's V contingency coefficients. Threshold of statistical significance for all the tests was set at $\mathrm{p}<0.05$.

The analyzed groups did not differ significantly in terms of their age, gynecological and obstetrical characteristics.

\section{RESULTS}

ESR, WBC and serum CRP in patients with/without specific IgA and IgG. The proportion of patients were determined with abnormal values of serum CRP, ESR and WBC, who tested positively for specific IgA and IgG. Elevated ESR was observed in 63\% of women testing positively for antiChlamydia trachomatis IgA (group B+D, $\mathrm{n}=144$ ); this fraction was significantly higher than the proportion of seronegative women with abnormal IgA titers (47\%, group $A+C, n=41)$. The presence of specific IgG was not associated with more frequent incidence of elevated ESR. Women with elevated IgA titers presented with leukocytosis significantly more often than those without ( $44 \%$, group $\mathrm{B}+\mathrm{D}$ vs. $26 \%$, group $\mathrm{A}+\mathrm{C}$ ). The largest proportion of patients with leukocytosis (52\%) was found in the group testing positively for both $\operatorname{IgA}$ and IgG (group D). Although elevated concentrations of serum CRP were observed more often in patients testing positively for IgA $(55 \%$, group $\mathrm{B}+\mathrm{D})$ than in those with normal titers of these antibodies ( $40 \%$, group $\mathrm{A}+\mathrm{C})$, this difference turned out to be insignificant. Also, the seroprevalence of antiChlamydia trachomatis IgG was not associated with more frequent occurrence of elevated serum CRP.

Results of diagnostic laparoscopy in patients with/without specific IgA (Fig. 1 and 2). Group A included a markedly larger proportion of patients without any laparoscopic pathologies (70\%) than groups B, D (both below 10\%) and C (less than $20 \%)$. Laparoscopic evidence of pelvic fluid collections and hyperaemia was significantly more often found in patients who tested positively for anti-Chlamydia trachomatis IgA $(60 \%$ - group B+D) than in seronegative women $(25 \%-$ group $\mathrm{A}+\mathrm{C})$. Women with and without elevated IgA titers did not differ significantly in terms of the occurrence of pelvic adhesions $(58 \%$ - group $\mathrm{B}+\mathrm{D}$ vs. $63 \%$ - group $\mathrm{A}+\mathrm{C})$ and incidence of appendicitis ( $8 \%$ - group $\mathrm{B}+\mathrm{D}$ vs. $16 \%$ - group $\mathrm{A}+\mathrm{C})$. The number of patients with laparoscopic evidence of retroperitoneal endometriosis was too small to conduct a statistical analysis. One patient who presented with adhesions to liver was eventually diagnosed with Fitz-Hugh-Curtis syndrome. This patient had elevated titers of both IgA and IgG against Chlamydia trachomatis (group D).

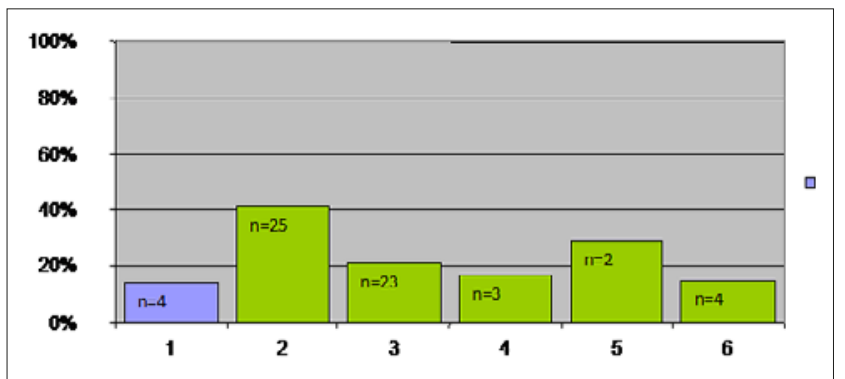

Figure 1.Distribution of patients with anti-Chlamydia trachomatis $\lg \mathrm{A}$ (group B+D) according to laparoscopic findings.

1 - normal laparoscopic presentation; 2 - pelvic fluid collections and hyperaemia within the minor pelvis; 3 - pelvic adhesions; 4 - retroperitoneal endometriosis; 5 - ectopic pregnancy; 6 - appendicitis.

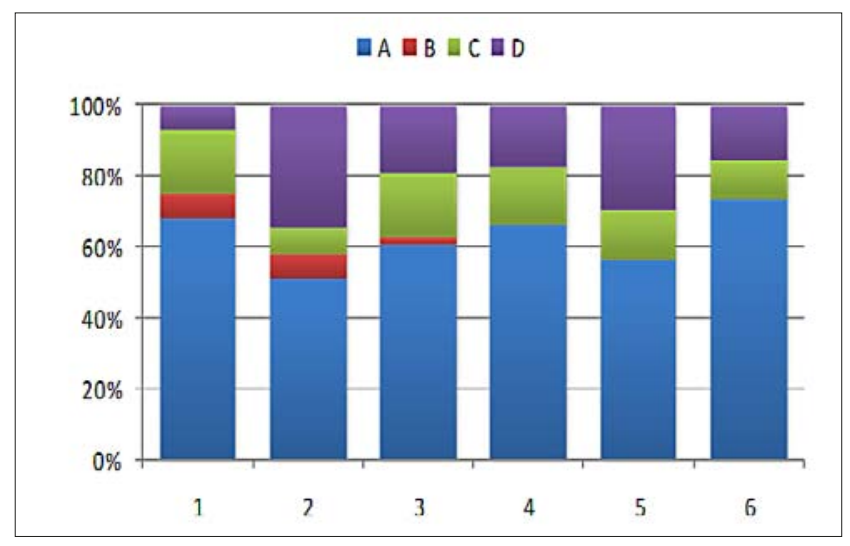

Figure 2. Distribution of patients from groups $A, B, C$ and $D$ according to laparoscopic findings.

1 - normal laparoscopic presentation; 2 - pelvic fluid collections and hyperaemia within the minor pelvis; 3 - pelvic adhesions; 4 - retroperitoneal endometriosis; 5 - ectopic pregnancy; 6 - appendicitis.

\section{DISCUSSION}

Elevated titers of anti-Chlamydia trachomatis antibodies were found in 70 (39\%) women from the examined group. This proportion was larger than reported previously by Bremer et al. $[13,14]$; according to these authors, chlamydiasis was the most common sexually-transmitted disease, present in $25 \%$ of their sample. Contrary to the presented study, the prevalence of chlamydiasiscorrelated with the age of the examined subjects; up to $64.3 \%$ of patients participating in this study were not older than 26 years $[13,14]$.

The current analysis was based on serological testing. However, to-date, no consensus has been reached with regards to the preferred method to detect Chlamydia trachomatis infection. According to Ngeow [15], the use of serological testing should be limited solely to the cases in which cervical swabs cannot be obtained. Rabenau et al. [16] analyzed the results of cervical swab examination and serological testing in 314 women; while only a weak correlation was found between the presence of active chlamydial infection and positive result of cervical swab examination, there was a strong association between the occurrence of infection and positive result of the serological test. Similar findings were published by Morre et al. [17], who determined the consistency rate of cervical swab examination and serological testing in 1,368 patients; the rate of Chlamydia trachomatis detection established solely on the basis of cervical swab examination was twice lower than in the case of serological testing. The study by Bułhak- 
Kozioł et al. [18] among patients with chlamydial cervicitis also provided arguments in favour of serological testing.

In all patients in the presented study, final diagnosis was established on the basis of laparoscopic examination performed within twenty-four hours of admission. Laparoscopy is an invasive procedure requiring general anaesthesia, posing a risk of surgical complications and representing a considerable economic burden. Nevertheless, according to Soper [19], laparoscopy can be beneficial even in patients with an already established clinical diagnosis, as it allows objective assessment of the severity of the disease, and thus to optimize its treatment. According to HenrySuchet and Tesquier [20] and Eschenbach et al. [21], early surgical intervention may result in lower morbidity. Recently, however, this approach is gaining a growing number of opponents. The authors of a meta-analysis published in 2008 showed that early diagnostic laparoscopy does not improve prognosis in patients with lower abdominal pain [22]. Consequently, many researchers opt for the development of an alternative algorithm based on laboratory, serological and microbiological findings, or even behavioural factors, in order to avoid laparoscopy in at least some proportion of cases $[23,24]$.

Aside from serological testing in the current study, ESR, serum CRP and WBC were also determined in the patients. Significant relationships were found between the presence of abnormal ESR, CRP and WBC values and elevated titers of anti-Chlamydia trachomatis IgA, as well as an associations between an increase in serum CRP, presence of elevated IgA and IgG titers and PID. Association between these laboratory parameters and PID was previously reported by other researchers [25]. Peipert et al. [26] observed elevated ESR in $70 \%$ of patients with lower abdominal pain, increased serum CRP in $71 \%$ and leukocytosis in 57\%. However, according to Bevan et al. [27], contrary to other sexually-transmitted diseases, e.g. gonorrhea, Chlamydia trachomatis infection is associated with only a slight increase in ESR, normal concentration of CRP and lack of leukocytosis. Tikkanen et al. [28] did not find a significant association between serum CRP and presence of anti-Chlamydia trachomatis antibodies. Similar findings were also reported by Karinen et al. [29].

The result of laparoscopic examination was normal in $15 \%$ of women participating in the presented study, and most of these patients (86\%) did not show elevated titers of anti-Chlamydia trachomatis antibodies. The most common laparoscopic pathology were pelvic adhesions, observed in up to $65 \%$ of the examined patients. The large fraction of women presenting with pelvic adhesions had elevated titers of anti-Chlamydia trachomatis IgG. This observation is consistent with the data published by other authors [30, 31]. Only one patient had adhesions to the liver and was eventually diagnosed with the Fitz-Hugh-Curtis syndrome. This woman had elevated titers of both IgA and IgG against Chlamydia trachomatis. According to the literature, 30$40 \%$ of patients may develop pelvic adhesions as an effect of immune response to Chlamydia trachomatis infection, resultant endothelial injury and irreversible changes, such as cicatrization and fibrosis [32]. Bevan et al. [27] found pelvic adhesions in $33.7 \%$ of patients with serological evidence of Chlamydia trachomatis infection. In the study conducted by Dabekausen et al. [33], up to 39\% of patients with pelvic pain had anti-Chlamydia trachomatis antibodies; pelvic adhesions were found in $35 \%$ of the seropositive women and in only $21 \%$ of the seronegative patients. Crha et al. [34] found pelvic adhesions in $39 \%$ of laparoscopically examined patients; up to $39.5 \%$ of these women tested positively for anti-Chlamydia trachomatis IgG. This constitutes another proof for an association between Chlamydia trachomatis infection and development of pelvic adhesions.

PID was the most common preliminary diagnosis made on admission of patients in the presented study. Laparoscopic evidence of pelvic inflammation was found in up to $60 \%$ of women with elevated titers of anti-Chlamydia trachomatis IgA and in only $25 \%$ of patients without. Even a stronger association between seroprevalence of anti-Chlamydia trachomatis antibodies and presence of PID was reported by Simms et al. [35]. According to these authors, up to $77 \%$ of patients with elevated titers of anti-Chlamydiatrachomatis IgA and IgG presented with adnexitis. Petersen et al. [32] also found serological evidence of chlamydiasis in 34.8\% of patients with PID. According to Vidhani et al. [36], serological testing should be a vital component of differential diagnosis in patients with suspected pelvic pathologies. The latter authors detected elevated titers of anti-Chlamydia trachomatis antibodies in up to $82.7 \%$ of their patients with laparoscopically confirmed PID [36]. In the study conducted by Puolakkainen et al. [37], chlamydiasis was eventually diagnosed in $73 \%$ of women with PID. The observations of the authors of the presented study and previously published data imply that laparoscopic evidence of pelvic fluid collections and hyperaemia, elevated WBC and ESR, correlate with the positive result of serological testing for Chlamydia trachomatis infection. This, in turn, suggests that laparoscopy could be avoided in at least some patients with evident clinical symptoms of PID, elevated WBC and ESR, and presence of specific IgA.

A potential association between Chlamydia trachomatis infection and endometriosis is a matter of ongoing discussion. Endometriosis was found in $9.7 \%$ of patients in the current study. According to Kligman et al. [38], up to $10 \%$ of women with endometriosis may test positively for anti-Chlamydia trachomatis antibodies. Higher prevalence of endometriosis among patients with past or present history of Chlamydia trachomatis infection was also reported by Heinonen and Leinonen [39]. However, in the study conducted by Debattista et al. [30], endometriosis was present in $29 \%$ and $40.5 \%$ of seropositive and seronegative patients, respectively.

Another important issue is the risk of ectopic pregnancy resulting from the formation of post-inflammatory adhesions within the uterine tubes of patients with chlamydiasis. Sharma et al. [40] found anti-Chlamydia trachomatis IgG in up to $50 \%$ of women with a history of ectopic pregnancy. The current study included seven patients in whom ectopic pregnancy was eventually identified as the cause of lower abdominal pain. The proportion of ectopic pregnancies in the subset of patients testing positively for anti-Chlamydia trachomatis IgA was slightly higher than in those without serological evidence the infection ( $4.88 \%$ vs. $3.47 \%)$.

\section{CONCLUSIONS}

Serological examination for Chlamydia trachomatis is helpful in the evaluation of patients with suspected pelvic infection, adding considerably to the results of laboratory testing (ESR, $\mathrm{CRP}, \mathrm{WBC})$. Elevated titers of anti-Chlamydia trachomatis 
antibodies are frequently associated with laparoscopic evidence of pelvic adhesions and inflammation.

\section{REFERENCES}

1. Won HR, Abbott J. Optimal management of chronic cyclical pelvic pain: An evidence-based and pragmatic approach. Int J Womens Health. 2010; 2: 263-277.

2. Malhotra M, Sood S, Mukherjee A, Muralidhar S, Bala M. Genital chlamydia trachomatis: An update. Indian J Med Res. 2013; 138(3): 303-316.

3. Papp J, Schachter J, Gaydos C, Van Der Pol B. Recommendations for the laboratory-based detection of chlamydia trachomatis and neisseria gonorrhoeae--2014. MMWR Recomm Rep. 2014; 63(RR-02): 1-19.

4. WHO. Global prevalence and incidence of selected curable sexually transmitted diseases: Overview and estimates. Geneva, World Health Organization, 2011.

5. Ceovic R, Gulin SJ. Lymphogranuloma venereum: Diagnostic and treatment challenges. Infect Drug Resist. 2015; 8: 39-47.

6. Price MJ, Ades AE, De Angelis D, Welton NJ, Macleod J, Soldan K, et al. Risk of pelvic inflammatory disease following chlamydia trachomatis infection: Analysis of prospective studies with a multistate model. Am J Epidemiol. 2013; 178(3): 484-492.

7. Marrazzo JM, Cates W. Interventions to prevent sexually transmitted infections, including hiv infection. Clin Infect Dis. 2011; 53(3).

8. Rours GI, Duijts L, Moll HA, Arends LR, de Groot R, Jaddoe VW, et al. Chlamydia trachomatis infection during pregnancy associated with preterm delivery: A population-based prospective cohort study. Eur J Epidemiol. 2011; 26(6): 493-502.

9. Banz C, Diedrich K, Hornung D. Antiflammatorische therapie bei endometriose. Gynäkologische Endokrinologie. 2004; 4: 223-225.

10. Jang HY, Burbelo PD, Chae YS, Kim T, Cho Y, Park HT. Nontuberculous mycobacterial infection in a clinical presentation of fitz-hugh-curtis syndrome: A case report with multigene diagnostic approach. BMC Womens Health. 2014; 14(95): 1472-6874.

11. Lehtinen M, Ault KA, Lyytikainen E, Dillner J, Garland SM, Ferris DG, et al. Chlamydia trachomatis infection and risk of cervical intraepithelial neoplasia. Sex Transm Infect. 2011; 87(5): 372-376.

12. Carter JD, Gerard HC, Whittum-Hudson JA, Hudson AP. Combination antibiotics for the treatment of chlamydia-induced reactive arthritis: Is a cure in sight? Int J Clin Rheumtol. 2011; 6(3): 333-345.

13. Bremer V. Sexuell übertragene erkrankungen in deutschland - stille epidemie. Deutsches Arzteblatt. 2005; 102: 2020-2023.

14. Bremer V, Hoffman A, Hamouda O. Epidemiologie der chlamydia trachomatis infektionen. Der Hautarzt. 2007; 58: 18-23.

15. Ngeow YF. Limitations of serodiagnosis in chlamydial genital tract infections. Ann Acad Med Singapore. 1996; 25(2): 300-304.

16. Rabenau HF, Kohler E, Peters M, Doerr HW, Weber B. Low correlation of serology with detection of chlamydia trachomatis by ligase chain reaction and antigen eia. Infection. 2000; 28(2): 97-102.

17. Morre SA, Munk C, Persson K, Kruger-Kjaer S, van Dijk R, Meijer CJ, et al. Comparison of three commercially available peptide-based immunoglobulin $\mathrm{g}$ (igg) and iga assays to microimmunofluorescence assay for detection of chlamydia trachomatis antibodies. J Clin Microbiol. 2002; 40(2): 584-587.

18. Bulhak-Koziol V, Zdrodowska-Stefanow B, Ostaszewska-Puchalska I, Mackowiak-Matejczyk B, Pietrewicz TM, Wilkowska-Trojniel M. Prevalence of chlamydia trachomatis infection in women with cervical lesions. Adv Med Sci. 2007; 52: 179-181.

19. Soper DE. Diagnosis and laparoscopic grading of acute salpingitis. Am J Obstet Gynecol. 1991; 164(5 Pt 2): 1370-1376.

20. Henry-Suchet J, Tesquier L. Role of laparoscopy in the management of pelvic adhesions and pelvic sepsis. Baillieres Clin Obstet Gynaecol. 1994; 8(4): 759-772.

21. Eschenbach DA, Wolner-Hanssen P, Hawes SE, Pavletic A, Paavonen J, Holmes KK. Acute pelvic inflammatory disease: Associations of clinical and laboratory findings with laparoscopic findings. Obstet Gynecol. 1997; 89(2): 184-192.

22. Maggio AQ, Reece-Smith AM, Tang TY, Sadat U, Walsh SR. Early laparoscopy versus active observation in acute abdominal pain: Systematic review and meta-analysis. Int J Surg. 2008; 6(5): 400-403.

23. Miettinen AK, Heinonen PK, Laippala P, Paavonen J. Test performance of erythrocyte sedimentation rate and c-reactive protein in assessing the severity of acute pelvic inflammatory disease. Am J Obstet Gynecol. 1993; 169(5): 1143-1149.

24. Nelson DB, Ness RB, Peipert JF, Soper DE, Amortegui AJ, Gluck J, et al. Factors predicting upper genital tract inflammation among women with lower genital tract infection. J Womens Health. 1998; 7(8): 1033-1040.

25. Hall MN, Leach L, Beck E. Clinical inquiries. Which blood tests are most helpful in evaluating pelvic inflammatory disease? J Fam Pract. 2004; 53(4): 330-331.

26. Peipert JF, Boardman L, Hogan JW, Sung J, Mayer KH. Laboratory evaluation of acute upper genital tract infection. Obstet Gynecol. 1996; 87(5 Pt 1): 730-736

27. Bevan CD, Johal BJ, Mumtaz G, Ridgway GL, Siddle NC. Clinical, laparoscopic and microbiological findings in acute salpingitis: Report on a united kingdom cohort. Br J Obstet Gynaecol. 1995; 102(5): 407-414.

28. Tikkanen M, Surcel HM, Bloigu A, Nuutila M, Hiilesmaa V, Ylikorkala $\mathrm{O}$, et al. Prediction of placental abruption by testing for c-reactive protein and chlamydial antibody levels in early pregnancy. Bjog. 2008; 115(4): 486-491.

29. Karinen L, Pouta A, Bloigu A, Koskela P, Paldanius M, Leinonen M, et al. Serum c-reactive protein and chlamydia trachomatis antibodies in preterm delivery. Obstet Gynecol. 2005; 106(1): 73-80.

30. Debattista J, Gazzard CM, Wood RN, Allan JA, Allan JM, Scarman A, et al. Interaction of microbiology and pathology in women undergoing investigations for infertility. Infect Dis Obstet Gynecol. 2004; 12(3-4): 135-145.

31. Ng EH, Tang OS, Ho PC. Measurement of serum ca-125 concentrations does not improve the value of chlamydia trachomatis antibody in predicting tubal pathology at laparoscopy. Hum Reprod. 2001; 16(4): 775-779.

32. Petersen EE, Clad A, Pichlmeier U, Bottcher M. The extended chlamydia trachomatis diagnosis in patients with pelvic inflammatory disease--a better approach for the diagnosis of upper genital tract infections. Clin Lab. 2003; 49(5-6): 277-281.

33. Dabekausen YA, Evers JL, Land JA, Stals FS. Chlamydia trachomatis antibody testing is more accurate than hysterosalpingography in predicting tubal factor infertility. Fertil Steril. 1994; 61(5): 833-837.

34. Crha I, Pospisil L, Stroblova H, Ventruba P, Zakova J, Huser M. [antibodies against the chlamydial heat shock protein in women with periadnexal adhesions]. Ceska Gynekol. 2006; 71(2): 127-131.

35. Simms I, Eastick K, Mallinson H, Thomas K, Gokhale R, Hay P, et al. Associations between mycoplasma genitalium, chlamydia trachomatis, and pelvic inflammatory disease. Sex Transm Infect. 2003; 79(2): 154-156.

36. Vidhani S, Mehta S, Bhalla P, Bhalla R, Sharma VK, Batra S. Seroprevalence of chalmydia trachomatis infection amongst patients with pelvic inflammatory diseases and infertility. J Commun Dis. 2005; 37(3): 233-238.

37. Puolakkainen M, Vesterinen E, Purola E, Saikku P, Paavonen J. Persistence of chlamydial antibodies after pelvic inflammatory disease. J Clin Microbiol. 1986; 23(5): 924-928.

38. Kligman I, Grifo JA, Witkin SS. Expression of the $60 \mathrm{kda}$ heat shock protein in peritoneal fluids from women with endometriosis: Implications for endometriosis-associated infertility. Hum Reprod. 1996; 11(12): 2736-2738.

39. Heinonen PK, Leinonen M. Fecundity and morbidity following acute pelvic inflammatory disease treated with doxycycline and metronidazole. Arch Gynecol Obstet. 2003; 268(4): 284-288.

40. Sharma K, Aggarwal A, Arora U. Seroprevalence of chlamydia trachomatis in women with bad obstetric history and infertility. Indian J Med Sci. 2002; 56(5): 216-217. 